\title{
Los 16 años de Fundación La Fuente'
}

\author{
CLAUDIOARAVENAGATICA \\ PontificiaUniversidad Católica de Chile \\ Chile \\ caravena@flf.cl
}

(Recibido:I3-09-20I6; aceptado: 20-09-20I6)

Hace un par de semanas recibí la invitación para escribir acerca de la fundación a la que pertenezco. No en vano llevo 15 años trabajando en ella, período que me hace acreedor tácito de ser guardián de la memoria, a veces frágil y otras, selectiva. Sin duda muchos de quienes abran las páginas de esta prestigiosa revista y se aventuren a conocer más acerca de nuestra institución, se irán poco a poco introduciendo en el mundo cultural yeducacional de mi país: Chile.

Escribo este texto en septiembre, un mes simbólico para nuestra historia reciente: el quiebre político más profundo se produjo este mes, la dictadura de Pinochet y sus alcances nos sobreviven, y han llevado estos últimos años a un despertar de la ciudadanía en contra de sus más profundas reformas: la educación, el sistema de pensiones, el libre mercado, la Constitución Política de 1980. En definitiva, las bases que sustentan la nueva sociedad chilena. ¿Qué tiene que ver este acercamiento con nuestra fundación? En forma aparente nada; pero al hacer solo unos rasguños en nuestra historia podemos identificar el verdadero motivo por el que nace y crece nuestra institución: la desigualdad.

Hace i6 años Verónica Abud, su marido y sus hijos crearon esta institución. Ella, profesora de educación básica, máster en educación en Estados Unidos y con una carrera de diez años en otra fundación ligada a la educación, decide crear su propio organismo, fruto de un cambio de vida. El objetivo inicial de esta nueva institución sería trabajar en el ámbito educacional de sectores vulnerables, aplicando programas de fortalecimiento en las áreas de lenguaje, matemáticas, arte y ciencias.

\footnotetext{
I Para citar este artículo: Aravena Gatica, Claudio (20i6). Los i6 años de Fundación La Fuente. Álabe 14. [www.revistaalabe. com]

DOI: Io.I5645/Alabe20i6.I4.IO
} 
Para llevar a cabo su objetivo, Verónica pondría en práctica lo aprendido en su máster: conseguir financiamiento privado para apoyar escuelas públicas en sectores de vulnerabilidad social y económica. Y así comenzó con los primeros atisbos de su programa Creando los lectores del mañana (CLM), el que desde un inicio buscó fortalecer el área de lenguaje gracias a una metodología que privilegiara el placer por la lectura, es decir, el programa buscaba -mediante la donación de una biblioteca atractiva, con buenos títulos, un espacio confortable y una bibliotecaria preparada- que los niños de estas escuelas no leyeran obligados un plan lector, sino que tuvieran estanterías abiertas llenas de nuevos títulos, principalmente cuentos ilustrados, para fortalecer la imaginación y quisieran así leer más libros cada vez.

\section{Un crecimiento sostenido}

Lo que a simple vista, con ojos del 20I6, puede sonar evidente, hace dieciséis años en nuestro país no lo era. A pesar de que a principios de los años go la reforma educacional promulgada por el gobierno de Eduardo Frei R-T eliminó el concepto de lectura obligatoria impuesto por Pinochet -el que seleccionaba títulos para fortalecer el espíritu patrio en el currículo educacional- las prácticas reiteradas se fueron modificando muy lentamente en las aulas. Los niños chilenos seguían leyendo años tras años los mismos títulos que sus hermanos mayores o que sus padres.

Poco a poco el espíritu rupturista (o chascón, o despeinado) del programan CLM iba tomando fuerza. El año 2OOI, la Fundación Andes y el Ministerio de Educación fueron promoviendo que la fundación llegara a nuevas escuelas: en los sectores de Colina y Cerro Navia en la capital; en Concón, Puchuncaví y Quillota, en la región de Valparaíso; en Puquillay bajo, en la zona campesina. Con esa avalancha de nuevos proyectos las otras áreas iniciales de la fundación fueron quedando de lado: el equipo de Verónica - en su mayoría profesionales jóvenes recién titulados de educación y letras- le dijo adiós a los programas de matemáticas, de ciencias y de las artes. Aunque no por mucho tiempo.

Solo pasaron dos años para constituirse en una fundación de promoción del libro y la lectura, la única en ese momento con esas características en Chile. A los programas de bibliotecas escolares, se sumaron tímidamente en un inicio - pero con gran impactolos bibliomóviles en zonas rurales: furgones, jeeps o camionetas cargadas con libros que visitaban escuelas alejadas de zonas urbanas, llevando libros a niños que en ese momento no contaban con material actualizado para poder leer. Gracias a una alianza estratégica con la Fundación Educacional Arauco -dependiente de la empresa forestal del mismo nombre- estas bibliomóviles fueron creciendo hasta transformarse en una flota de más de 45 en todo Chile que, aun pasados más de diez años de su creación, siguen funcio- 
nando apoyados por los fondos económicos que el Estado entrega a través del Fondo del Libro, que pertenece al Consejo Nacional de la Cultura y las Artes.

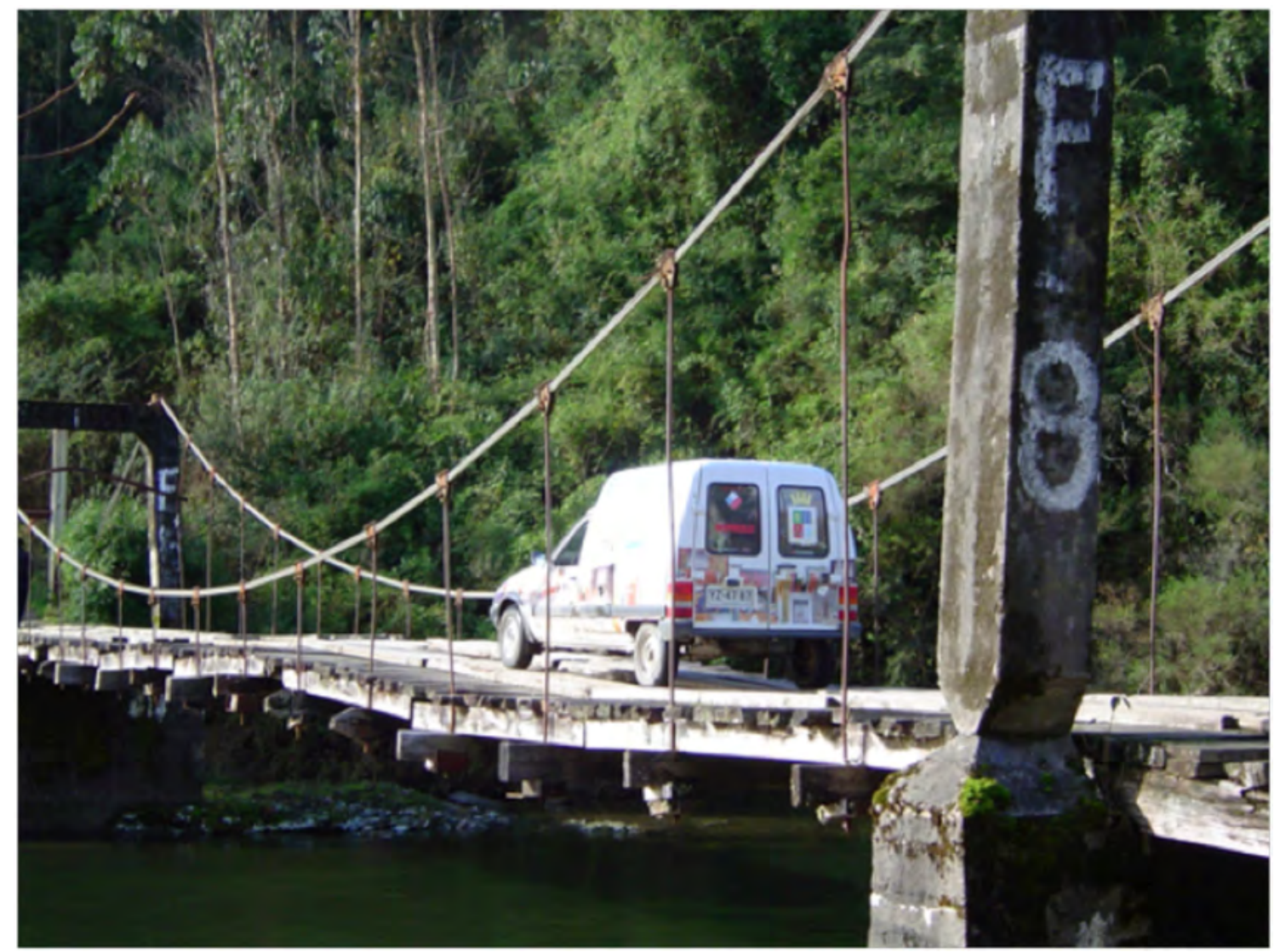

Bibliomóvil Rural

El crecimiento de la fundación se produjo a partir del año 2003 cuando la administración de los centros comerciales Mall Plaza le propusieron a Verónica la creación de un proyecto innovador: bibliotecas públicas dentro de un mall. En Chile, el crecimiento de los años go trajo consigo el fortalecimiento del modelo norteamericano, las tarjetas de crédito, los shopping centers, las grandes tiendas de retail. No fue raro entonces que este modelo, que se iniciara en los sectores de ingresos altos, se fuera expandiendo a los nuevos barrios de clase media emergente; y las personas vieran con alegría y entusiasmo cómo un gran páramo era reemplazado por una mole de tiendas, servicios, comida y diversión. Ya en el 2000 -y tomando prestado el concepto al sociólogo chileno Tomás Moulian- el mall se convirtió en la nueva plaza pública; y en ella nació Biblioteca Viva.

La primera biblioteca se abrió el año 2003, en un local de 450 metros cuadrados. El programa fue íntegramente financiado por la administración de Mall Plaza haciendo uso de un mecanismo creado por el Estado chileno: la Ley de Donaciones Culturales, entidad diseñada por el senador Gabriel Valdés para incentivar que las empresas privadas puedan invertir en proyectos del ámbito cultural descontando hasta el $50 \%$ de lo entrega- 
do a entidades culturales, en impuestos. Haciendo uso de los datos del Informe de Gestión 2015 de este proyecto -disponible en el sitio web www.bibliotecaviva.cl-podemos señalar que nuestras sedes tuvieron durante el año pasado: 895.854 visitas anuales, 2I.283 personas inscritas, IOI.OOO asistentes a actividades culturales, I64.3I2 libros prestados. Estas bibliotecas, como todo espacio actual, no solo ofrece servicios de préstamos de material bibliográfico, también son un espacio de trabajo conjunto, de talleres, cursos, exposiciones, son un espacio de encuentro comunitario para niños y jóvenes; y una posibilidad clara de acceso cultural en comunas que tienen carencia de estas acciones.

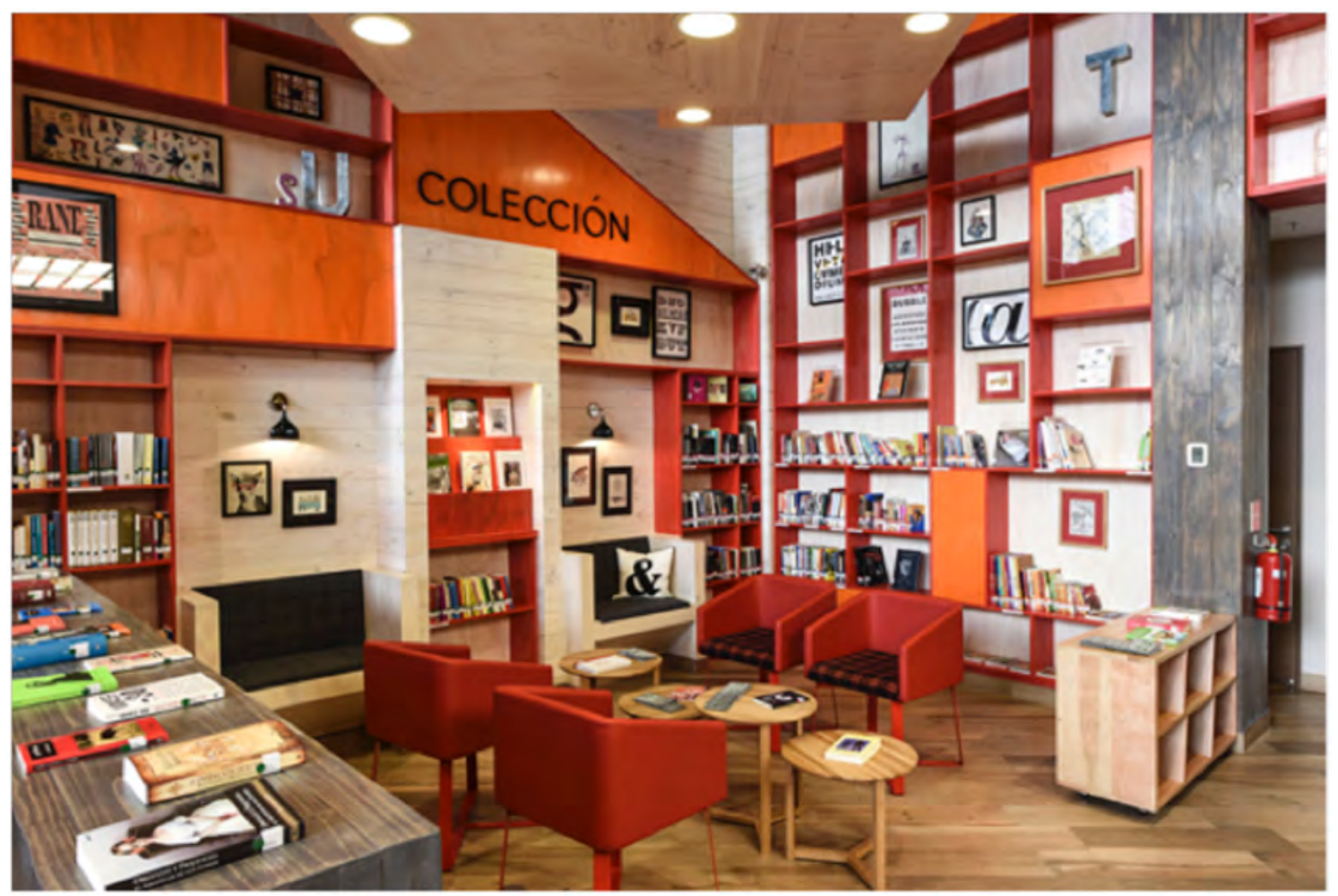

Biblioteca Viva

Otro ámbito de colaboración con el sector público, junto con la instalación de bibliotecas en escuelas y bibliomóviles, es el programa de apoyo a bibliotecas públicas dependiente del SNBP (Sistema Nacional de Bibliotecas Pública de la Dirección de Bibliotecas Archivos y Museos, Dibam). Este programa, creado en el año 2OOI, también con financiamiento de la Fundación Andes, busca poder colaborar con el sistema público allegando recursos en equipamiento, habilitación, capacitación y hace un par de años, en construcción de nuevas bibliotecas. Durante estos años, con financiamiento privado y la Ley de Donaciones Culturales, hemos trabajado en Cerro Navia, Puchuncaví, Peñalolén, Yungay, San José de la Mariquina, Constitución y Arauco.

En estas dos últimas comunas hemos diseñado proyectos emblemáticos, producto de la recuperación de equipamiento cultural que nace producto del terremoto y mare- 
moto (grado 8.8, escala Richter) del 27 de febrero de 20IO. En ambas ocasiones en reuniones con la comunidad, se dio origen a un proyecto conjunto que reunió a los vecinos, las agrupaciones culturales locales, a las autoridades municipales y las empresas privadas en la construcción de nuevos proyectos. En Constitución se diseñó una nueva biblioteca que toma como base la madera, propia actividad industrial de la zona. El proyecto, inaugurado en noviembre de 20I5, ya ha recibido reconocimiento en Canadá y ahora postula a los prestigiosos premios RIBA (Royal Institute of British Architects). Por su parte, en Arauco, también se recogieron los intereses de la comunidad indígena, quienes destacaron la presencia cercana a la futura biblioteca del cerro Colo-Colo, lugar fundacional de la cultura mapuche en la zona. El proyecto de corte contemporáneo rescató vistas y elementos de estas comunidades, además de congregar en un espacio de I.40o metros cuadrados una biblioteca pública, un teatro municipal, salas de trabajo y ensayo y un gran patio comunitario. Este proyecto es el más ambicioso llevado a cabo por la fundación en su historia.

\section{Nuevos aires}

El crecimiento y la versatilidad de los proyectos desarrollados -todos en el ámbito de los libros y la lectura-trajeron consigo cambios y desafíos, la fundación tuvo que constituirse en una gran entidad, con I2o trabajadores, preocupada por la formación de sus empleados, como también de quienes reciben los proyectos que se implementan. El trabajo en investigación y formación empieza a ser clave en el funcionamiento de los proyectos: el año 2006 se inicia el proceso con la creación de la encuesta nacional Chile y los libros, que midió el avance de hábitos lectores cada dos años hasta el 20IO, cuando el Estado - a través del Consejo Nacional de la Cultura y las Artes- se hace cargo de tener una encuesta similar. Se suman a estas encuestas investigaciones en jóvenes (Familia y Escuela: su influencia en la formación para los lectores del mañana) y niños (Los niños y los libros; Esto no es un cuento, volúmenes 1 y 2). Todas disponibles en el sitio web de la fundación (www.fundacionlafuente.cl).

En el período 2OIO-20I5, junto a la Facultad de Educación de la Pontificia Universidad Católica de Chile creamos el primer diplomado online en Chile sobre literatura infantil, juvenil y promoción del libro y la lectura, el diplomado FLIJ capacitó en estas áreas a más de 500 interesados en realizar mediación de lectura o desarrollarse en el ámbito de la edición de libros. Junto a la Facultad de Educación de la Universidad Diego Portales, creamos el proyecto Biblioteca-laboratorio LEA / profesor Roberto Cabrera, un proyecto piloto que busca insertar una biblioteca escolar dentro de una facultad de educación para que los estudiantes tengan contacto directo con las novedades editoriales recientes y puedan en ella - gracias a una alianza con los jardines infantiles y escuelas básicas cercanas-hacer animaciones lectores a niños. 
El año 2OI5 nace el Centro de Estudios Troquel como otra forma de fortalecer el trabajo realizado por la fundación. Este centro tiene por objetivos diseñar e implementar programas de capacitación para profesores, bibliotecarios y mediadores de lectura en general, en formato presencial y online. Los cursos, de bajo costo, son desarrollados por profesionales en literatura, edición, ilustración, animación lectora (primera infancia, niños y jóvenes), narrativa gráfica, gestión de bibliotecas, entre otras áreas. Junto a eso, el Centro Troquel tiene a su cargo el Comité de valoración de libros infantiles y juveniles, un organismo formado por I7 profesionales de la fundación, de distintos proyectos y de distintas partes del país, quienes tienen la misión de leer todos los libros editados durante el año. Cada semestre, las recomendaciones de Imprescindibles son agrupadas en un boletín que se distribuye vía digital e impresa, en forma abierta; y son comprados para las distintas bibliotecas de la fundación para que queden a disposición del público interesado.

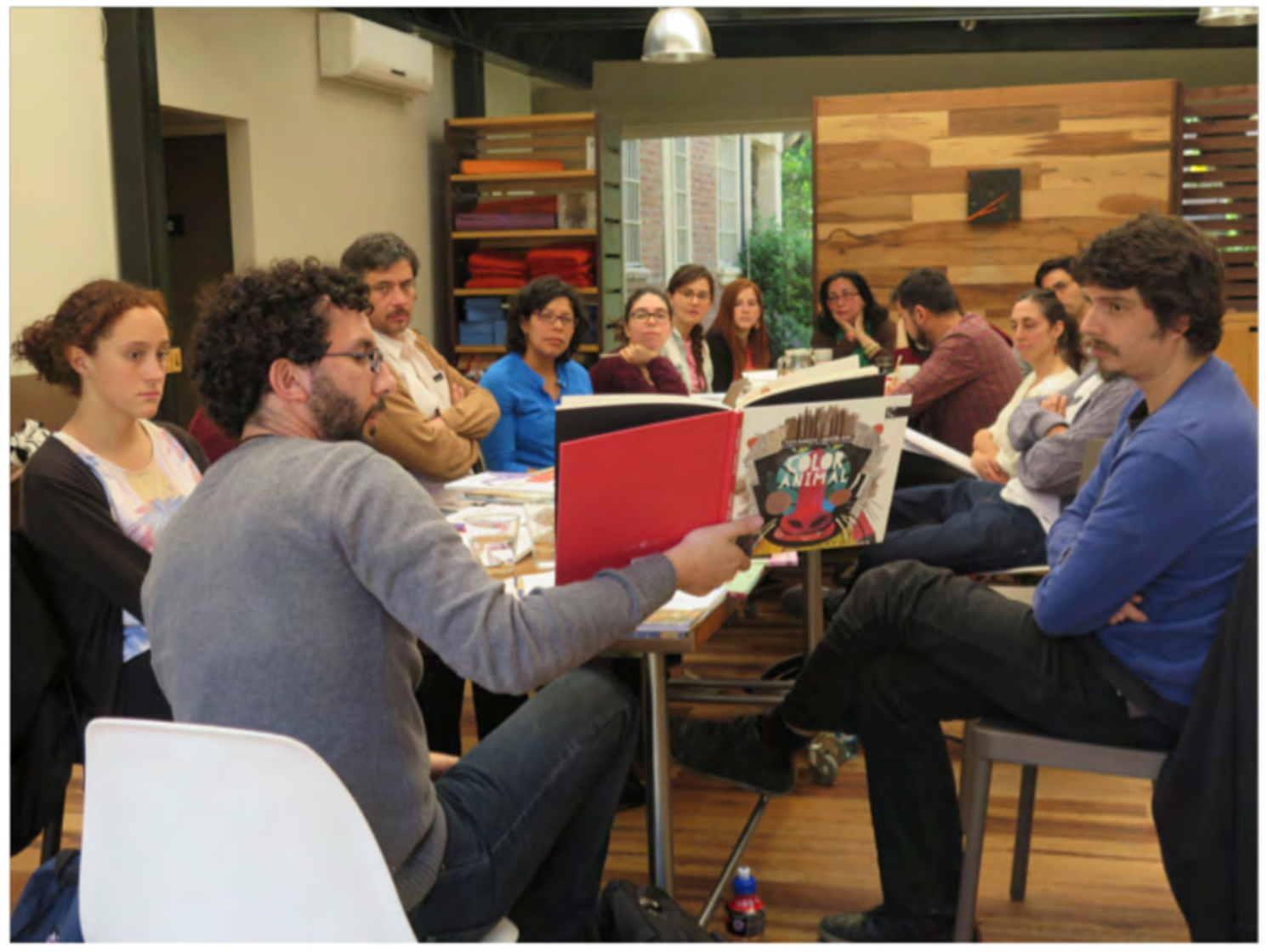

Sesión comité de valoración de libros Troquel 
La irrupción de las redes sociales nos puso como desafío sumar contenidos a estos mecanismos de difusión infinita. Así los objetivos impulsados por la fundación se han podido extender más allá de nuestras fronteras - mar y cordillera- que a veces más que delimitar nos limitan. Facebook, twitter, nuestro sitio web, han incorporado entrevistas, artículos, columnas o reseñas escritas por miembros de nuestra institución o colaboradores externos. Nos hemos transformado en un medio de comunicación, que cada semana actualiza sus contenidos y los difunde para conocimiento general.

Decía al inicio que la memoria es frágil y a veces, selectiva. En estos i6 años son muchos los proyectos que no alcanzaron el éxito esperado, las ideas que quedaron escritas en alguna libreta, los informes que nadie leyó. Tampoco han sido parte de este recuento los portazos al presentar una idea, las bibliotecas cerradas, los "No" al revisar el presupuesto de un proyecto, las zancadillas siempre presentes. Es parte del crecimiento el caerse varias veces para triunfar en un proyecto. Durante estos años hemos sido parte de la historia de nuestro país, con su crecimiento desbordado y desigual, con sus demandas ciudadanas y protestas callejeras, con sus perspectivas de cambio. A pesar de esta convulsión, vemos con optimismo nuestro futuro y esa alegría es patente cada vez que un niño abre un libro, sonríe y se acomoda en un sofá, a disfrutar. 